
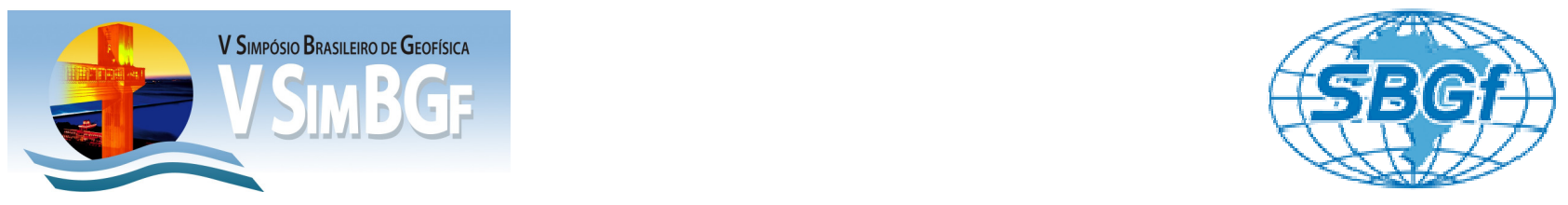

\title{
Gradiente Geotérmico do Município de Coronel Fabriciano no Estado de Minas Gerais
}

Jackeline Cabral Reis (jackeline.cabral@me.com) e Antonio Jorge de Lima Gomes (antonio.gomes@ufvim.edu.br) UFVJM - Universidade Federal dos Vales do Jequitinhonha e Mucuri

Copyright 2012, SBG - Sociedade Brasileira de Geofísica

Este texto foi preparado para a apresentação no V Simpósio Brasileiro de Geofísica, Salvador, 27 a 29 de novembro de 2012. Seu conteúdo foi revisado pelo Comitế Técnico do V SimBGf, mas não necessariamente representa a opinião da SBGf ou de seus associados. E proibida a reprodução total
propósitos comerciais sem prévia autorização da SBG.

\section{Resumo}

Como parte de um projeto de pesquisa iniciado em 2010 pelo laboratório de geotermia da UFVJM - Universidade Federal dos Vales Jequitinhonha e Mucuri, em fase de implementação, foi realizada uma compilação, reavaliação e análise das condições geotérmicas e geológicas do poço situado no Município de Coronel Fabriciano em Minas Gerais, com dados obtidos a partir de uma perfilagem térmica realizada em 1977. Este trabalho faz parte de um projeto de iniciação científica denominado: "Energia Geotérmica: Uma fonte alternativa nos Vales do Jequitinhonha e Mucuri", com apoio da Fapemig e do MEC. Foram utilizados os métodos CVL (convencional) e CBT (temperatura estável no fundo do poço) na determinação dos gradientes geotérmicos, cujos valores ficaram compreendidos entre 9,2 e $17,1^{\circ} \mathrm{C} / \mathrm{km}$, respectivamente, para os métodos CVL e CBT. Os valores obtidos indicam que a região é caracterizada por gradientes geotérmicos relativamente baixos característicos de regiões pré-cambrianas, sendo que, o tipo de formação geológica predominante, onde o poço está situado, pertence ao Complexo Mantiqueira.

\section{Introdução}

Com o objetivo de compreender o campo térmico regional do Município de Coronel Fabriciano, no Estado de Minas Gerais, foi realizada uma nova compilação dos dados de temperatura, obtidos na perfilagem térmica, realizada em 1977 por Hamza e sua equipe. O poço denominado de E-10 teve sua perfuração em Agosto de 1976 e sua perfilagem térmica foi realizada no ano seguinte em 04/06/1977.

Neste trabalho, reavaliamos e compilamos os resultados anteriores, e fizemos também uma nova análise do perfil térmico, do gradiente geotérmico e da geologia da região. A importância do conhecimento geológico permitiu inferir e interpretar melhor os resultados das temperaturas observadas no perfil térmico.

É característico em regiões pré-cambrianas e termicamente estáveis valores baixos de gradiente geotérmico (Hamza e Muñoz, 1996; Hamza et al, 2005; Hamza e Gomes, 2005; Gomes; 2009; Gomes et al, 2011; e Lopes et al, 2012).
O poço na qual foi realizada a perfilagem térmica, possui coordenadas geográficas de Latitude 19 $31^{\prime} 07^{\prime \prime} \mathrm{S}$ e longitude 42 37 ' 44" W.

Apresentamos a seguir na Figura (1) a localização regional do Município de Coronel Fabriciano, compreendido na porção leste do Estado de Minas Gerais.

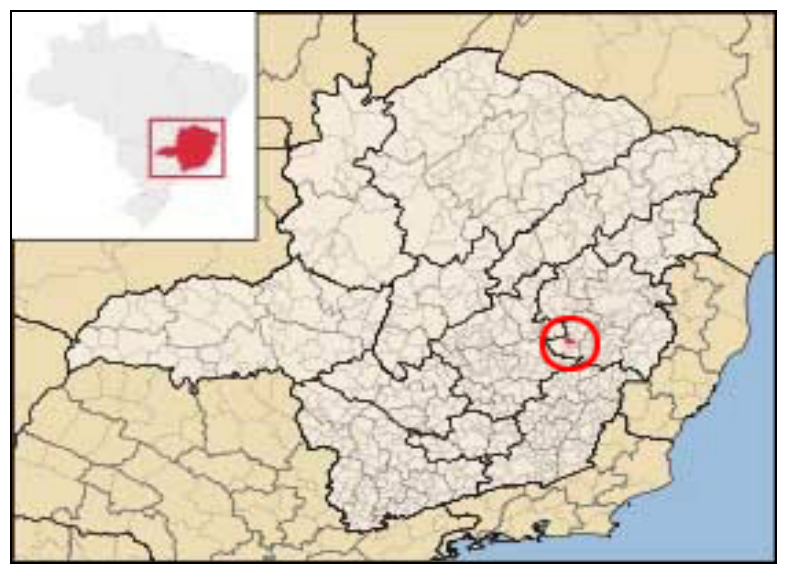

Figura 1 - Mapa regional com a localização do Município de Coronel Fabriciano em Minas Gerais.

\section{Geologia e Geofísica Regional}

$O$ poço E-10 onde foi realizada a perfilagem térmica, se encontra na formação geológica do Complexo Mantiqueira. Este complexo corresponde ao "Complexo Basal" descrito nas folhas Guanhães, Marilac e Santa Maria do Suaçuí (CPRM, 2000).

É composto por ortognaisses cálcio-alcalinos, de composição granítica, tonalítica, quartzo sienítica, monzonítica, quartzo monzonítica, monzodiorítica e diorítica, migmatizados ou não, com intercalações de anfibolito, metagabro/metadiorito e norito. Apresenta-se na Figura (2) o mapa geológico da região de Coronel Fabriciano (CPRM, 2000).

As formações geológicas predominantes da região de Coronel Fabriciano são o Complexo da Mantiqueira representado no mapa anterior por Am onde predominam Anfibólio-biotita gnaisse bandado com composição tonalítica, granodiorítica e granítica. Subordinam-se anfibolitos, migmatitos, gnaisses charnockíticos e biotititos (CPRM, 2000).

A partir de uma pesquisa realizada no sitio web do SIAGAS sobre a geologia regional, pode-se concluir que no local em que o poço se encontra, o tipo de formação geológica é do Complexo Mantiqueira, mas em poços 
próximos e distintos, observamos muitos solos de Aluviões. O solo denominado como Aluvião é composto por sedimentos inconsolidados arenosos, areno-argilosos com níveis de cascalho. (CPRM, 2000).

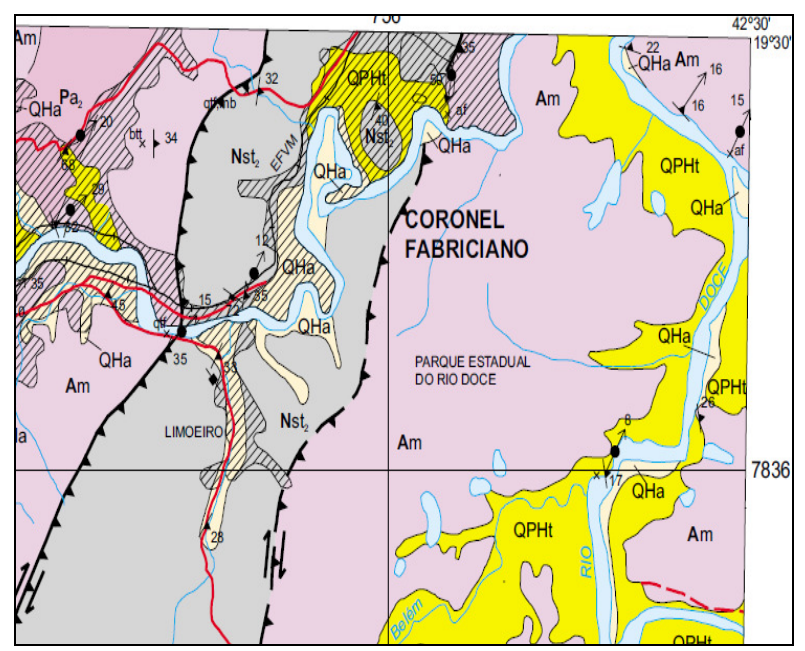

Figura 2 - Mapa geológico regional de Coronel Fabriciano em Minas Gerais. Am - Complexo Mantiqueira e Nst Formação São Tomé (CPRM,2000).

\section{Metodologia}

Para a determinação do gradiente geotérmico foram utilizados dois métodos distintos, que eram os mais propícios para as condições do local, que são o método convencional (CVL) e o de temperatura estável do fundo de poço (CBT).

A base para uma avaliação de recursos geotermais são as informações sobre estas temperaturas em subsuperfície. Temos dois conjuntos de dados sobre as temperaturas em profundidade: medidas diretas e estimativas indiretas (Bolditzar, 1958; Bullard, 1965; Fournier, 1991).

A temperatura do poço foi medida com a utilização de um termistor do tipo NTC, semicondutor que apresenta propriedade física específica onde a resistência elétrica diminui com um aumento de temperatura (Gomes, 2009). O método CVL (convencional) utilizado na determinação do gradiente geotérmico do poço consiste no primeiro passo para a interpretação do gradiente, onde selecionamos a escolha do intervalo de profundidade apropriado, que deve estar livre de qualquer perturbação que possa interferir o regime geotérmico local. Consideram-se as camadas geológicas lateralmente homogêneas, de propriedades térmicas constantes e que possuem dimensões físicas bem definidas em relação aos intervalos das medidas. O segundo passo é o método de ajuste linear aos dados de profundidade e temperatura obtidas nas perfilagens térmicas. O método dos mínimos quadrados permite estimar os valores dos coeficientes de ajuste linear.

No presente caso, para um conjunto de $\mathrm{N}$ pares de dados (zi, Ti) os coeficientes são o gradiente térmico $(\Gamma)$ e o intercepto $\left(T_{0}\right)$, dada por:

$$
\begin{gathered}
\Gamma=\frac{N \sum z_{i} T_{i}-\sum z_{i} \sum T_{i}}{N \sum z_{i}^{2}-\left(\sum z_{i}\right)^{2}} \\
T_{0}=\frac{\sum z_{i}^{2} \sum T_{i}-\sum z_{i} \sum z_{i} T_{i}}{N \sum z_{i}^{2}-\left(\sum z_{i}\right)^{2}}
\end{gathered}
$$

O método CBT (temperatura estável do fundo do poço) é utilizado quando se supõe que as variações térmicas geradas pelos movimentos dos fluidos tornam-se praticamente nulas na parte inferior do poço, então a temperatura estável do fundo do poço possa ser usada para determinação do gradiente geotérmico, desde que a temperatura de superfície média anual seja conhecida. A relação a seguir permite a determinação do gradiente CBT.

$$
T_{C B T}=T_{0}+q \sum_{i=1}^{N} R_{i} h_{i}
$$

Onde: $R i$ é a resistividade térmica da camada i. O termo somatório se refere à resistência térmica cumulativa das formações presentes até o fundo do poço onde foi efetuada a medida de temperatura.

\section{Resultados}

Os resultados dos gradientes geotérmicos obtidos com base nas temperaturas medidas experimentalmente no perfil térmico do poço $E-10$ serão descritos abaixo na Tabela (2).

Foi utilizada neste trabalho uma temperatura média anual da região, do ano em que foi realizada a perfilagem de $19^{\circ} \mathrm{C}$ aproximadamente, tais dados obtidos no Atlas Climatológico (Serra, 1955).

As temperaturas anteriores à década de 1950 do século passado ainda não se encontram perturbadas pelo aumento recente ocasionado pelo aquecimento global.

Tabela 2 - Valores obtidos do gradiente geotérmico por tipo de método utilizado e temperatura

\begin{tabular}{|c|c|c|}
\hline \multicolumn{3}{|c|}{$\begin{array}{c}\text { Síntese dos Resultados do } \\
\text { Gradiente Geotérmico }\end{array}$} \\
\hline Método & Ts (ㅇ) & G (ํ)/km) \\
\hline CVL & 19,1 & 9,2 \\
\hline CBT & 18,5 & 17,1 \\
\hline
\end{tabular}

O município encontra-se situado na região bioclimática, cujo clima é do tipo subtropical úmido (Golfari, 1975).

As temperatura de superfície utilizadas basearam-se no Atlas Climatológico do Brasil (Serra, 1955). 
Com base nas informações obtidas compilamos e obtivemos os resultados dos gradientes geotérmicos do poço E-10 com os métodos CVL e CBT, cujos resultados são apresentados na Figura (3) no final deste trabalho. Os valores encontrados na determinação do gradiente geotérmico foram de $9,2 \stackrel{\circ}{\circ} \mathrm{C} / \mathrm{km}$ pelo método $\mathrm{CVL}$ e também de $17,1^{\circ} \mathrm{C} / \mathrm{km}$ para o $\mathrm{CBT}$, cujos resultados estão graficamente descritos na Figura (3).

No gráfico da Figura (3) estão traçados os limites de temperatura com a equação correspondente do gradiente geotérmico e seu intercepto, além dos ajustes lineares correspondentes.

\section{Conclusões:}

Os valores obtidos dos gradientes geotérmicos no poço E-10 localizado no Município de Coronel Fabriciano pelos métodos CVL e CBT, se encontram respectivamente, num intervalo compreendido entre 9,2 e $17,1^{\circ} \mathrm{C} / \mathrm{km}$.

Os valores obtidos pelas análises mostraram que o local possui gradientes geotérmicos relativamente baixos.

Os valores resultantes pelos dois métodos utilizados na determinação do gradiente geotérmico estão relacionados com regiões pré-cambrianas, sobretudo, pelo local das medidas, que está situado na formação geológica do Complexo Mantiqueira.

Ressalta-se, no entanto, que o valor CVL encontra-se relativamente um pouco abaixo do esperado, já que nessas regiões, o gradiente térmico costuma estar compreendido entre 14 e $20^{\circ} \mathrm{C} / \mathrm{km}$.

A presença de solos aluvionares pemite que exista um fluxo hidráulico variável o que perturba as temperaturas do poço, as quais, na presença de águas meteóricas ou de nascentes, acabam apresentando valores menores do que o esperado.

Neste contexto identificou-se uma perturbação diminuindo a temperatura no local do poço. Em última hipótese um bombeamento recente do poço não justificado ou não oficializado.

A ausência de outras perfilagens no local impede um avanço do conhecimento geotérmico a nível local e regional. Neste sentido, precisamos de mais medidas de temperatura em subsuperfície, assim como mais levantamentos geotérmicos, para um estudo mais aprofundado da região.

\section{Agradecimentos:}

Agradecemos à equipe do Laboratório de Geotermia do Observatório Nacional/ MCT, que realizou a perfilagem. Em especial ao Doutor Valiya Manathal Hamza. Agradecemos a FAPEMIG e ao MEC pelos incentivos à iniciação científica.

\section{Referências bibliográficas:}

Bolditzar, 1958 T. Bolditzar, The distribution of temperatures in flowing wells. Am. J. Sci.256 pp.294-298.

Bullard, E. C., 1965, Historical introduction to terrestrial heat flow, American Geophysics Union, Geophys. Mon. Ser., in Lee, W. H. K. Terrestrial Heat Flow, v8 pp. 1-6.
CPRM - PROJETO LESTE, 2000. Mapeamento geológico e cadastramento de recursos minerais da região leste de Minas Gerais. Belo Horizonte SEME/COMIG/MME/CPRM.

Fournier, R. O., 1991, Water geothermometers applied to geothermal energy, ed. D'Amore F., UNITAR-UNDP, New York, 253-273.

Bizzi, L. A.; Schobbenhaus, C.R.; Vidotti M. e Gonçalves, J. H., 2003. Geologia, Tectônica e Recursos Minerais do Brasil CPRM, Brasília.

Golfari, L., 1975. Zoneamento ecológico do estado de Minas Gerais para reflorestamento. PNUD/FAO/IBDFBRA/71/545, 1975. 65p. (Série Técnica no 3).

Gomes, A.J.L. e Hamza, V.M., 2003 Avaliação de Recursos Geotermais do Estado do Rio de Janeiro. $8^{\circ}$ International Congress of the Brazilian Geophysical Society, Rio de Janeiro.

Gomes, A. J. L. ;Hamza, Valiya Mannathal. 2009. Avaliação de Recursos Geotermais da Bacia do Paraná. Tese de Doutorado, Observatório Nacional.

Gomes, A.J.L. e Hamza, V.M., 2009. Gradiente e Fluxo Geotérmico da Bacia do Paraná, Congress of the Brazilian Geophysical Society, Salvador, Brazil.

Gomes, A. J. L. and HAMZA, V.M., 2005. Gradiente e Fluxo Geotérmico do Estado de Santa Catarina, 9응 International Congress of the Brazilian Geophysical Society, 6p., Cd-rom, Salvador, Brasil.

Gomes, A.J.L. ; Alexandrino, C., H. . 2011. Mapeamento de Gradiente Geotérmico do Município de Teófilo Otoni no Estado de Minas Gerais. In: 12th International Congress Of The Brazilian Geophysical Society.

Hamza, V.M. and Muñoz, M., 1996. Heat Flow map of South America, Geothermics, V.I 25, n 6, pp. 599-646.

Hamza, V.M., Silva Dias, F.J.S., Gomes, A.J.L. and Terceros, Z.G.D., 2005. Numerical and Functional Representations of Regional Heat Flow in South America, Physics of the Earth and Planetary Interiors, Volume 152, 4, p.223-256.

Lopes, K.J. ; Silva, J.M.P., GOMES, A.J.L. 2012. Identificação de área de recarga com vistas à sustentabilidade ambiental com base em medidas geotérmicas em Teófilo Otoni. In: IX Congresso Nacional de Meio Ambiente, Poços de Caldas, Brasil.

Meister, E.M., 1973. Gradientes geotérmicos nas bacias sedimentares Brasileiras, B. T. Petrobrás, 16(4), 221-232.

Serra; Adalberto 1995 - Atlas Climatológico do Brasil (Vol. 1 - médias, extremos, totais). Cons. Nac. de Geografia e Serv. Meteorológicos, vl. 1. Rio de Janeiro.

SIAGAS - Sistema de informações de águas
subterrâneas. http://siagasweb.cprm.gov.br/layout/detalhe.php?ponto=3 100002150) Acesso em 23 de junho de 2012. 


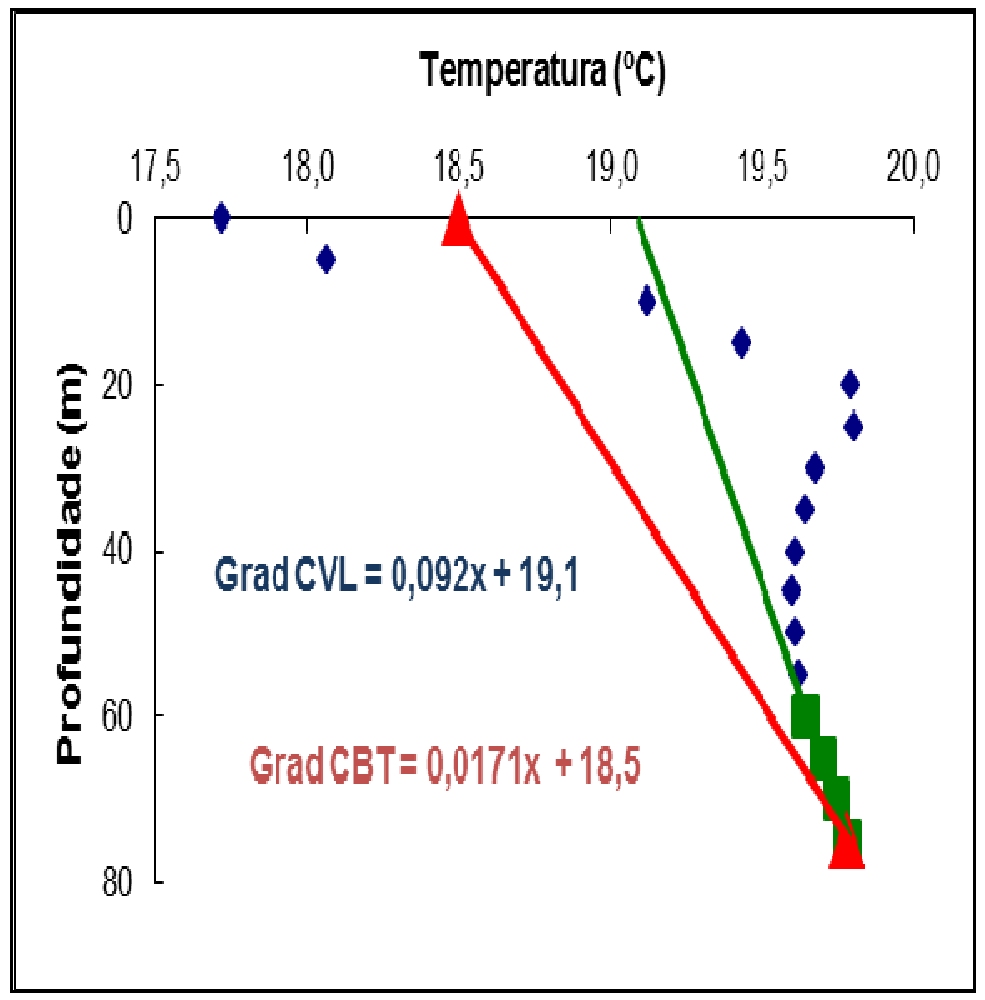

Figura 3- Nova avaliação do perfil térmico do poço E-10. 